\title{
The aceE involves in mycolic acid synthesis and biofilm formation in Mycobacterium smegmatis
}

\author{
Suting Chen, Tianlu Teng, Shuan Wen, Tingting Zhang and Hairong Huang*
}

\begin{abstract}
Background: The integrity of cell wall structure is highly significant for the in vivo survival of mycobacteria. We hypothesized that changes in morphology may indicate changes in cell wall metabolism and identified an aceE gene mutant (aceE-mut) which presented a deficient colony morphology on 7H10 agar by screening transposon mutagenesis in Mycolicibacterium smegmatis, basonym Mycobacterium smegmatis (M. smegmatis). This study aimed to identify the functional role of aceE gene in cell wall biosynthesis in M. smegmatis.

Results: We observed that the colony morphology of aceE-mut was quite different, smaller and smoother on the solid culture medium than the wild-type (WT) strain during the transposon library screening of $M$. smegmatis. Notably, in contrast with the WT, which aggregates and forms biofilm, the aceE-mut lost its ability of growing aggregately and biofilm formation, which are two very important features of mycobacteria. The morphological changes in the aceE-mut strain were further confirmed by electron microscopy which indicated smoother and thinner cell envelope images in contrast with the rough morphology of WT strains. Additionally, the aceE-mut was more fragile to acidic stress and exhibited a pronounced defects in entering the macrophages as compared to the WT. The analysis of mycolic acid (MA) using LC-MS indicated deficiency of alpha-MA and epoxy-MA in aceE-mut strain whereas complementation of the aceE-mut with a wild-type aceE gene restored the composition of MA.
\end{abstract}

Conclusions: Over all, this study indicates that aceE gene plays a significant role in the mycolic acid synthesis and affects the colony morphology, biofilm formation of M. smegmatis and bacteria invasion of macrophage.

Keywords: Mycobacterium smegmatis, aceE, Biofilm, Mycolic acid, Cell wall

\section{Background}

The mycobacterial cell wall is mainly composed of three types of macromolecules i.e. peptidoglycan, arabinogalactan and mycolic acids (specific components shared by the members of the order Corynebacterineae (e.g., mycobacteria, nocardia, and rhodococci)) [1]. Glycolipids, porins and lipoarabinomannan as well as its variants, which are anchored to the cell membrane by diacylglycerol, are also

\footnotetext{
*Correspondence: hhr_jys@163.com

National Clinical Laboratory on Tuberculosis, Beijing Key laboratory for Drug Resistant Tuberculosis Research, Beijing Chest Hospital, Capital Medical University, Beijing Tuberculosis and Thoracic Tumor Institute, Beijing 101149, China
}

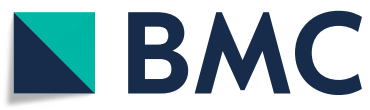

essential components [2]. The carboxyl group of mycolic acids is vertically covalently linked to the hydroxyl group of arabino-galactan by ester bond, arabino-galactan is linked to the peptidoglycan layer by phospholipid bond, whereas other glycolipids and free lipids are regularly distributed in the thicker layer of mycolic acids [3-5]. These collectively form a thick, dense, poorly permeable cell wall, which not only allows mycobacteria to resist the dry environment and harmful chemicals but also allows it to reproduce in the macrophages $[4,6]$. Therefore, the molecules involved in the cell wall biosynthesis of TB bacilli have been considered as important anti-TB drug targets. Among the existing anti-TB drugs, isoniazid,

(c) The Author(s). 2020 Open Access This article is licensed under a Creative Commons Attribution 4.0 International License, which permits use, sharing, adaptation, distribution and reproduction in any medium or format, as long as you give appropriate credit to the original author(s) and the source, provide a link to the Creative Commons licence, and indicate if changes were made. The images or other third party material in this article are included in the article's Creative Commons licence, unless indicated otherwise in a credit line to the material. If material is not included in the article's Creative Commons licence and your intended use is not permitted by statutory regulation or exceeds the permitted use, you will need to obtain permission directly from the copyright holder. To view a copy of this licence, visit http://creativecommons.org/licenses/by/4.0/ The Creative Commons Public Domain Dedication waiver (http://creativecommons.org/publicdomain/zero/1.0/) applies to the data made available in this article, unless otherwise stated in a credit line to the data. 
ethionamide and ethambutol target the cell wall synthesis pathways, among which isoniazid and ethambutol are the first-line anti-TB drugs.

Pyruvate dehydrogenase (PDH) is an enzyme complex that catalyzes the conversion of pyruvate into acetyl-coA in vivo. The complex mainly consists of three enzymes, which are respectively called E1, E2 and E3 components of PDH according to the order in which they participate in the reactions. Through a series of chemical reactions of pyruvate decarboxylation, the glycolytic pathway (the final product is pyruvate) and the tricarboxylic acid cycle (the initial reactant is acetyl-coA) can be effectively connected [7]. As an important intermediate metabolite, acetyl-coA not only participates in tricarboxylic acid cycle as well as the glyoxylate cycle but also provides carbon source for the synthesis of mycolic acid and lipids $[8,9]$. Studies have also found that the genes involved in gluconeogenic pathway and glyoxylate cycle are up-regulated in Mtb isolated from macrophages, mouse lung tissues and tissue samples of patients, suggesting that the compensatory metabolism of acetyl-coA is necessary for intracellular growth and persistence in vivo $[10,11]$.

In the present study, we found a mutant strain in which the aceE gene, encoding the E1 component of $\mathrm{PDH}$, was inactivated by Himar1 transposon insertion
(aceE-mut). This mutant had obvious differences in colony morphology (smaller plaque, edge smooth and round) and defects in biofilm formation in contrast with the wild-type (WT) strains. Further analyses indicated that the aceE gene deficiency affected the cell mycolic acid profile of M. smegmatis.

\section{Results}

\section{The aceE-Mut exhibited unusual colony morphology}

By screening the M. smegmatis transposon library, a transposon mutant showed obvious differences in colony morphology (small, smooth without jagged edges and yellow color) on agar plate when compared to the parental WT M. smegmatis strain (Fig. 1a). DNA sequencing and analysis of the MycoMar/M. smegmatis chromosomal junction revealed that the transposon mutant had an insertion at a TA dinucleotide within the aceE gene and the distance of the Tn insertion from the start codon of the aceE gene is $2243 \mathrm{bp}$ (Fig. $1 \mathrm{~b}$ and c). No apparent difference in growth rate was found between the WT and aceE-mut strain with the Middlebrook 7H9 neutral medium culturing (Fig. $2 \mathrm{a}$ and $\mathrm{b}$ ). However, we observed that the aceE-mut strain dispersed uniformly in the broth without Tween-80, while the WT $M$. smegmatis formed more clumps in the same medium. Notably, the growth rate of aceE-mut was significantly

A

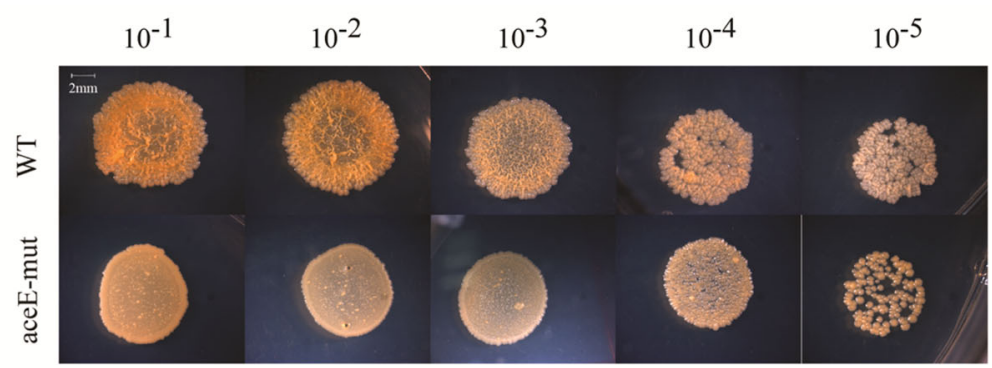

B

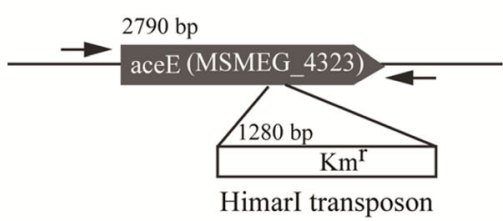

$\mathrm{C}$
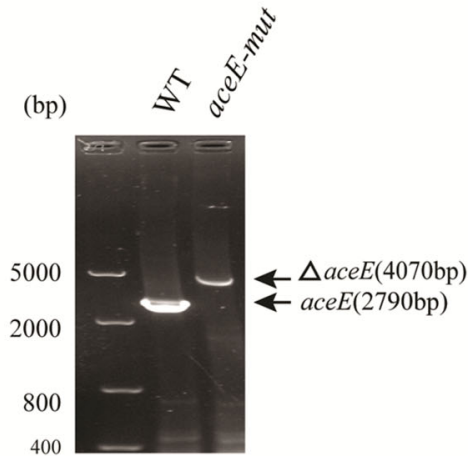

Fig. 1 The identification of M. smegmatis aceE-mut. a. The aceE-mut showed smoother colony morphology in contrast to WT. The culture of M. smegmatis $\mathrm{mc}^{2} 155$ and aceE-mut were $10 \times$ diluted and $10 \mu \mathrm{L}$ culture aliquots were spotted on the Middlebrook $7 \mathrm{H} 10$ medium supplemented with $0.2 \%$ glycerol. The images were taken after incubation at $37^{\circ} \mathrm{C}$ for 3 days on $7 \mathrm{H} 10$ plates. b. Himarl transposon insertion site in aceE gene; $\mathbf{c}$. PCR verification of the aceE transposon mutant. WT: M. smegmatis $\mathrm{mc}^{2} 155$; aceE-mut: aceE gene deficiency mutant selected from M. smegmatis $\mathrm{mc}^{2} 155$ transposon library 


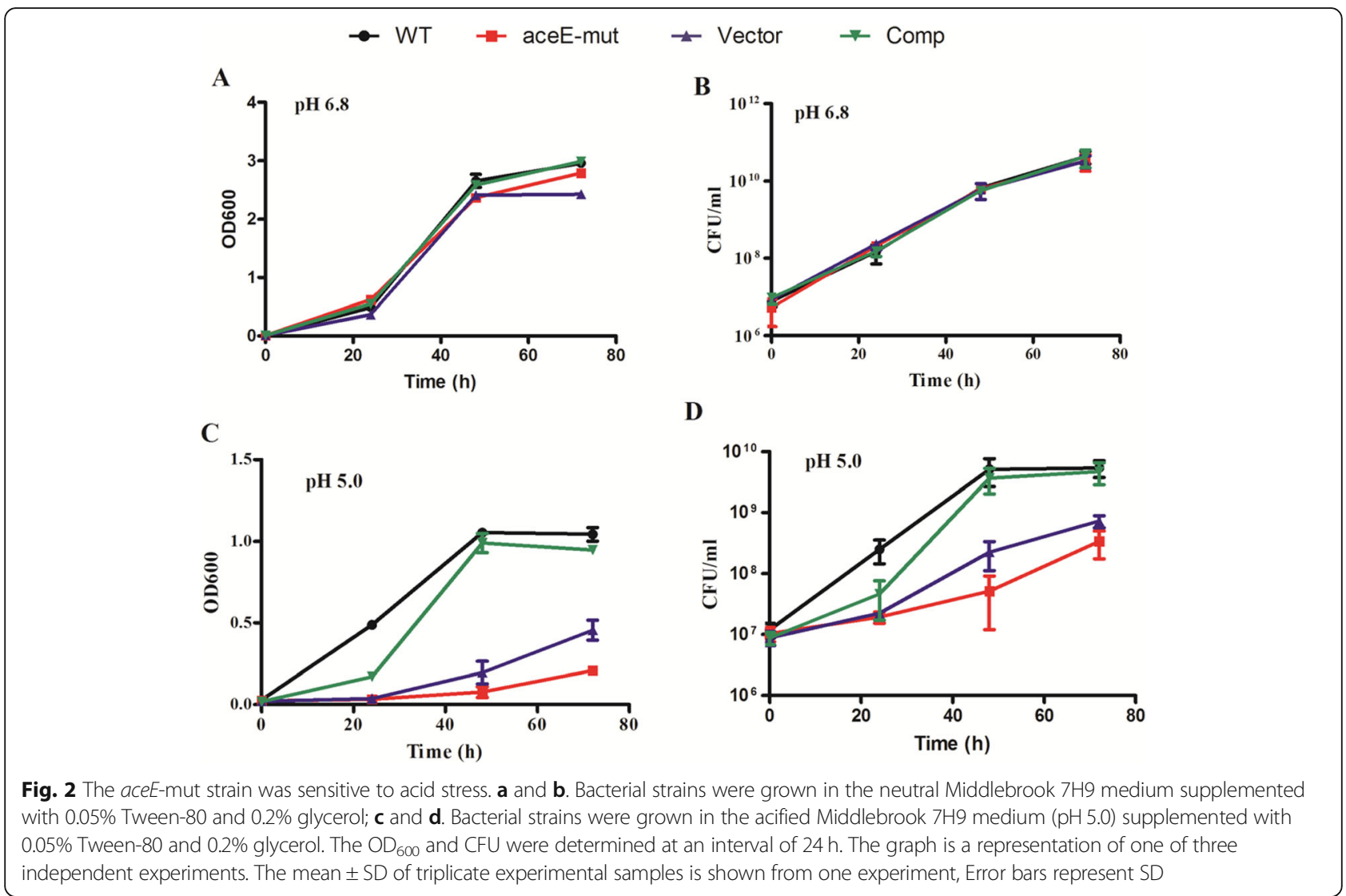

lower than the WT strain in acidified 7H9 media during logarithmic and stationary phases (Fig. 2c and d). Comp strain demonstrated similar growth dynamics as WT in both culture conditions. These results demonstrated that aceE gene is dispensable in $M$. smegmatis and the disruption of aceE gene in $M$. smegmatis renders the bacteria more sensitive to acid stress, and this feature could be complemented with the wild-type copy of aceE gene.

\section{Pellicle and biofilm formation defected in aceE-Mut}

In contrast to the significant pellicle growth that appeared on the air-liquid interface in the WT strain culture, pellicle was absent from the aceE-mut strain culture when grown in 7H9 medium without Tween80 supplement and shaking (Fig. 3a). In order to better quantify the biofilm formation defects in aceE-mut, strains were cultivated in M63-based liquid medium and biofilm formation was analyzed visibly as well as using the crystal violet assay. Consistently, aceE-mut did not form biofilm in the M63-based liquid medium either. Notably, the complementary expression of aceE gene in aceE-mut strain restored the pellicle and biofilm formation to the WT levels (Fig. 3b). Taken together, these results indicated that aceE gene is involved in pellicle and biofilm formations.
aceE gene affected the cell surface morphology and cell wall architecture of $M$. smegmatis

The surface morphology of WT and aceE-mut was observed by scanning electron microscopy. The aceE-mut cells were slightly slender than WT cells, and their surfaces were smoother than WT cells (Fig. 4). These microscopic observations were consistent with the smooth phenotype on agar plate. The cell morphology and cell wall architecture were further examined by transmission electron microscopy, which showed that the cell wall of the mutant was thinner $(P<0.01)$ than WT and Comp strains (Fig. 5).

\section{Cell wall permeability analysis}

In order to analyze the effect of disruption of the $a c e E$ gene on cellular response against stress conditions in vitro, the aceE-mut and WT strains were treated with commonly used anti-TB drugs and several antimicrobial agents in vitro. No significant differences in antimicrobial sensitivity tests between WT and mutant strains were observed (Supplementary Table S1 and Supplementary Fig. S1).

\section{aceE-Mut has a defect in invasion of macrophages}

The role of aceE in cell wall integrity and the M. smegmatis resistance against acidic environment suggests that it may also protect the bacilli from microbicidal activity 
A
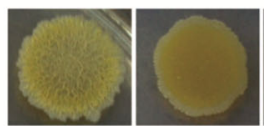
WT

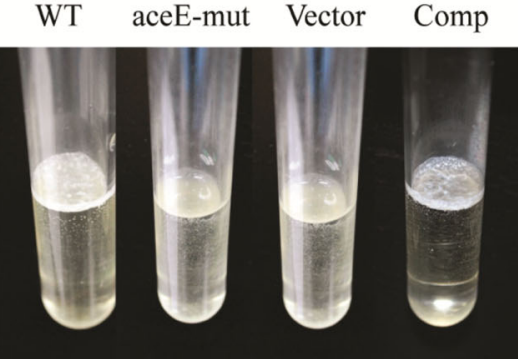

B

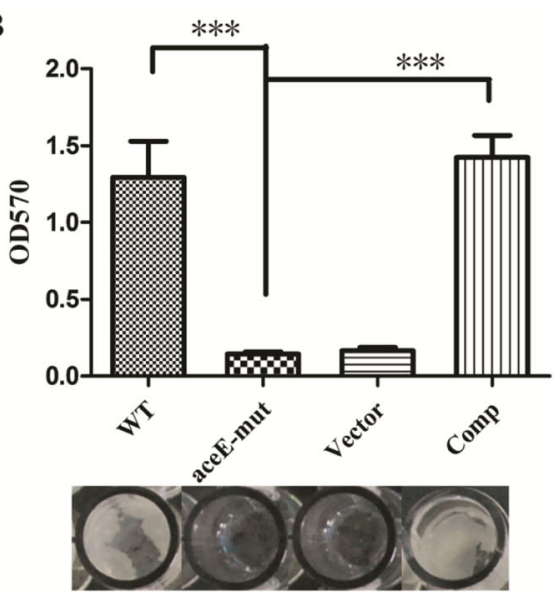

Fig. 3 The effect of aceE deficiency on pellicle and biofilm formation of mycobacteria. $\mathbf{a}$. aceE-mut is defective in pellicle formation, while the complementary expression of aceE gene in aceE-mut stain can recover the formation of pellicle in $7 \mathrm{H} 9$ medium without Tween-80 in standing culture. Each experiment performed in triplicate. b. Quantification of the biofilm formation after crystal violet staining. Mean optical density for five biological replicates per strain \pm SD for a representative experiment from 3 experiments is shown. Significant differences were determined by One Way ANOVA and are indicated by ${ }^{* * *}(P<0.001)$. Error bars represent SD

of macrophages. Therefore, we investigated and compared the impact of aceE inactivation on invasion and intracellular survival of mutants with its corresponding complemented strain (Comp) and WT strain. As shown in Fig. 6, aceE-mut strains and its parent strain showed different abilities to invade THP-1 macrophages after $2 \mathrm{~h}$ of incubation. aceE-mut exhibited pronounced defects in entering the macrophages as compared to the WT and Comp strains. A significant difference between the mutant and WT strains of approximately $0.5 \log$ unit was observed at $\mathrm{t}=0 \mathrm{~h}$ post-infection $(P<0.05$ at $\mathrm{MOI}=1: 1$; $P<0.01$ at $\mathrm{MOI}=1: 10)$. However, CFU of the mutant infection group was not lower than the WT infection group at $\mathrm{t}=24 \mathrm{~h}$.

\section{Disruption of aceE gene affected the mycolic acid composition}

The mycolic acid $\left(\mathrm{C}_{60} \sim \mathrm{C}_{90}\right)$ composition was analyzed by HPLC using Mycobacteria Identification System. In contrast to WT strain, the mutant strain possessed higher proportion of short-chain mycolic acids but lower proportion of long-chain mycolic acids (Supplementary Fig. S2). The percentage of mycolic acid with equivalent carbon length of 5 to 8 in the mutant strain was significantly lower than the wild strain $(P<0.05)$, suggesting a potential role of aceE gene in mycolic acid metabolism. A further LC-MS-based systematic analysis of mycolic acid and lipids did not identify any obvious differences in the phospholipid and glycolipid compositions $(P>$ 0.05 ) between the WT and aceE-mut strains (Fig. 7a), whereas certain kinds of $\alpha$-mycolic acids were deficient in aceE-mut strains $(P<0.05)$ and these changes were fully restored upon complementation (Fig. $7 \mathrm{~b}$ and c).

\section{Discussion}

Transposon mutagenesis has been used extensively as a useful tool for studying gene function of mycobacteria. In this study, we identified an aceE gene mutant presenting deficient colony morphology on 7H10 agar using transposon mutagenesis method. Hence, it is reasonable to speculate defective cell wall biosynthesis in the mutant. Changes in the structure of cell envelope components may affect the normal physiological metabolic processes in mycobacteria including, transportation of antimicrobial agents across the plasma membrane and mycobacterial survival in stress conditions, etc.

Previous studies have shown that mycobacterial PDH genes (aceE, dlaT and lpd) are not only involved in pyruvate metabolism, but may also have more complex biological functions [12-16]. It has been observed that dlaT gene knockout significantly affects the in vitro growth of TB bacilli in the standard medium. The mutant is not only sensitive to reactive nitrogen intermediates but its virulence is also reduced in the infected mice. In addition, the study found that dla $T$ inhibitors can selectively kill non-self-replicating TB bacilli, suggesting that dlaT gene may be associated with latent $\mathrm{TB}$ infection in host cells $[12,14,15]$. Study also showed that the disruption of lpd gene in Mtb leads to decreased virulence with lower bacterial load in lung and other organs in infected mice. Other study revealed that the LPD is a component of branched chain ketones acid dehydrogenase, which takes part in succinyl CoA metabolism of amino acids such as valine (Val), isoleucine (Ile) [16].

However, the biological function of the E1 component of mycobacterial PDH remains poorly understood. In 2005, Tian et al., confirmed for the first time that $a c e E$ is 

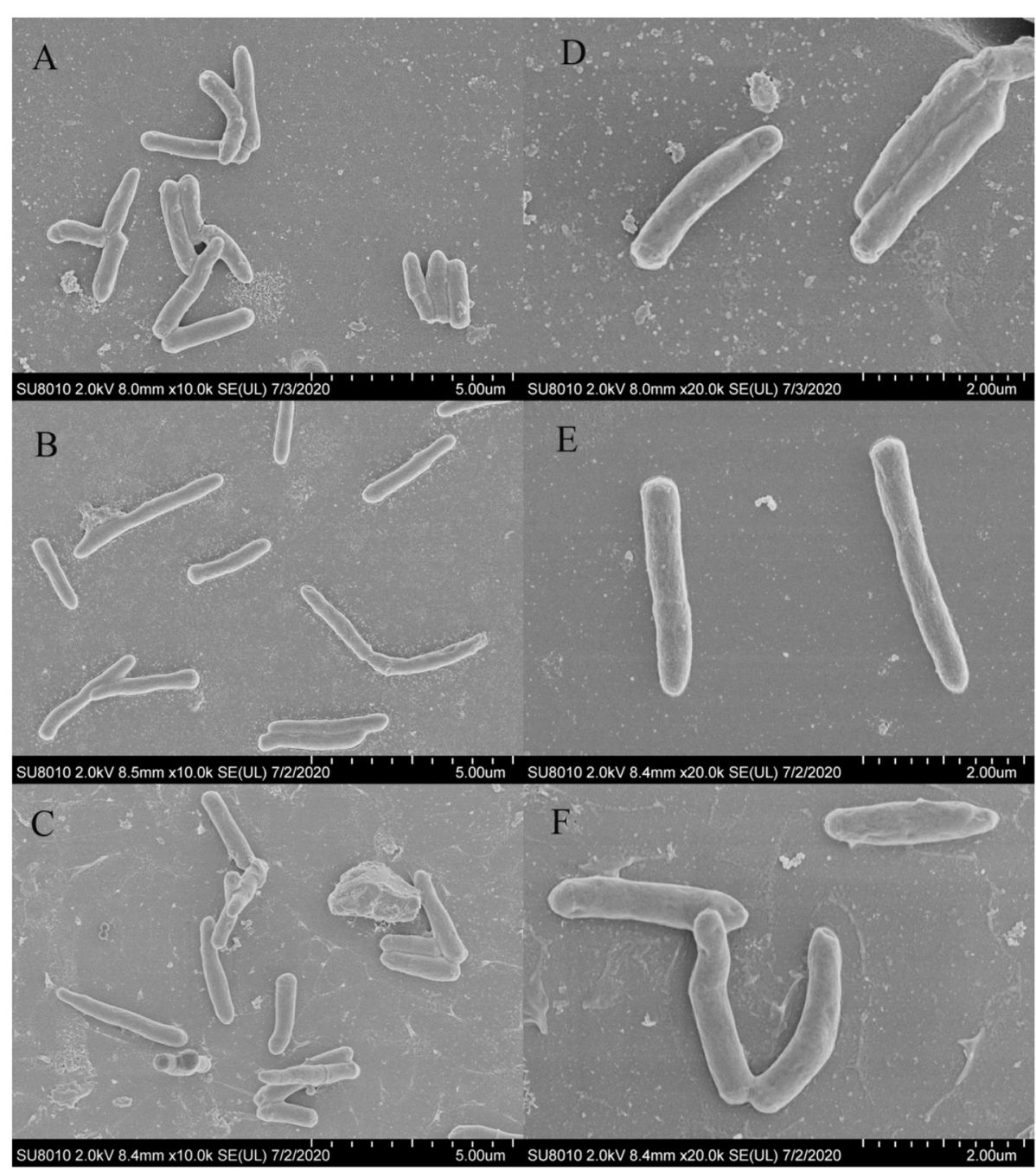

Fig. 4 The morphology of M. smegmatis $\mathrm{mc}^{2} 155$ ( $\mathbf{a}$ and $\mathbf{d}$ ), aceE-mut (b and $\mathbf{e}$ ) and Comp (c and $\mathbf{f}$ ) under SEM. Bars represent $5 \mu \mathrm{m}(\mathbf{a}, \mathbf{b}$ and $\mathbf{c}$ ) and $2 \mu \mathrm{m}$ (d, e and $\mathbf{f})$

the gene encoding the E1 component of PDH complex in Mtb [17]. In 2008, Li et al., found that the expression level of aceE gene in H37Rv was significantly higher than that of H37Ra during the course of macrophages infection [18]. This result is consistent with the results of gene chip analysis conducted by Manganelli et al., in 2001 [19]. Earlier studies had shown that H37Rv respiration is stronger than H37Ra. Although both, virulent $\mathrm{H} 37 \mathrm{Rv}$ and the non-virulent H37Ra strains, rely on glycolysis and aerobic respiration for glucose metabolism, it is believed that glucose metabolism in $\mathrm{H} 37 \mathrm{Rv}$ may be more dependent on the glycolytic pathway $[20,21]$. Therefore, it is speculated that the up-regulated expression of aceE gene enables it to not only participate in the aerobic respiration as a component of $\mathrm{PDH}$, but also guarantees successful glycolysis under the hypoxic conditions and thus provides excellent energy supply for growth of the virulent $\mathrm{H} 37 \mathrm{Rv}$ strain. Another study showed that AceE component of PDH forms a fourcomponent peroxidase system with DlaT/AhpD/AhpC, which assists the reductase action using pyruvate as a source of electrons [22], and thus protect the pathogen by participating in antioxidant of antinitraxidative defense. All of the above studies suggest that aceE gene plays an important role in Mtb metabolism in vitro and in vivo.

In the present study, we screened the random transposon mutants and found that the inactivation of aceE gene affects the colony morphology and biofilm formation in M. smegmatis which suggests its potential to affect the lipid metabolism and cell wall biosynthesis. The findings obtained in our study are in agreement with Viswanathan et al. [23]. Integrity of the cell wall has important biological significance for the in vitro and in vivo survival of bacteria. To further investigate the characteristics of the aceE-mut, series of assays were 

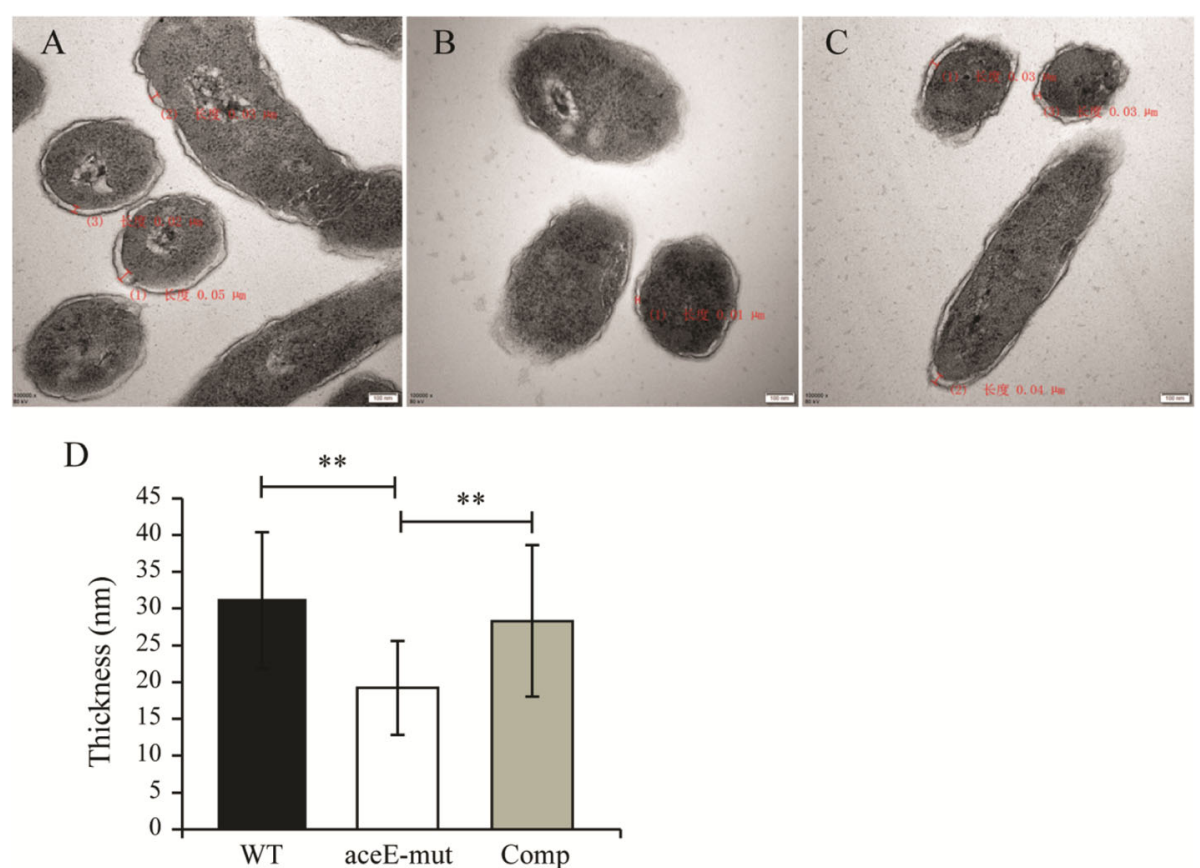

Fig. 5 The cell wall thickness of M. smegmatis $\mathrm{mc}^{2} 155$ (a), aceE-mut (b) and Comp (c). D. The quantification of the cell wall thickness for each strain, one hundred mycobacteria in the visual field were randomly selected. The largest distance between the outer membrane and the inner membrane of each cell was measured and statistically analyzed. Bars represent $100 \mathrm{~nm}(\mathbf{a}, \mathbf{b}$ and $\mathbf{c})$. Histogram bars in panel (d) indicate standard deviation. Significant differences were determined by One Way ANOVA and are indicated by ** $(P<0.01)$

performed to compare the phenotypes of aceE-mut with the WT strains. We further analyzed the key composition of mycolic acids in each strain and identified altered composition in the aceE-mut which suggests that this gene may be involved in the metabolism of mycolic acid in mycobacteria. Therefore, it was necessary to thoroughly analyze the cell wall composition of both WT and aceE-mut strains and explore the role of aceE gene in the process of cell wall synthesis and metabolism. Comparative analysis of the lipid and mycolic acid profiles of the $M$. smegmatis aceE-mut, $M$. smegmatis WT and the Comp using LC-MS indicated that the metabolism of certain alpha-MA and epoxy-MA was deficient in aceE-mut, which demonstrated that the role of $a c e E$ was associated with mycolic acids, but not with other fatty acids found in $M$. smegmatis. In addition, we observed that the aceE-mut possess a distinct cell morphology and ultrastructural appearance compared with the WT strain when grown in broth culture, that may result from the inability of the mutants to synthesize certain kinds of alpha-MA. Thus, the inactivation of aceE also impacts bacterial physiology that ranges from reduced biofilm formation to changes in the cell morphology and cell wall thickness. Consistent with other studies, our study also found that the loss of long chain mycolic acids or oxygenated mycolic acids effects biofilm formation [24-26]. Previous study by Trivedi et al., showed that $\mathrm{H} 37 \mathrm{Rv}$ are of marginally smaller size during biofilm formation, however, it was difficult to compare the differences in cell size between the aceE-mut and WT strains in our study because of the morphological differences between these two strains (aceE-mut strains is more slender than WT strains). Most importantly, aceEmut was more susceptible to acidic environments than the parental M. smegmatis, suggesting plausible role of aceE gene in stress tolerance inside the host.

aceE gene is more readily expressed in the virulent Mtb H37Rv (than H37Ra) throughout the course of infection, that not only suggests its important role in the virulence, survival and persistence of Mtb, but also makes this gene a potential target for the development of newer vaccines and anti-TB drugs. In M. smegmatis, the full length of the MSMEG4323 (aceE) gene is 2790 bp. Using blast tools, aceE gene sequence of M. smegmatis was analyzed which highlighted its conservation in $M$. smegmatis and a similarity of $82 \%$ with the aceE gene in Mtb. As a model bacterium widely used in the study of functional genes of mycobacterium, M. smegmatis can also be used to investigate the potential role of aceE gene in mycobacterial cell wall biosynthesis. In the present study, the stress assay demonstrated that aceE gene helped mycobacteria to withstand acidic stress environment that also suggests its plausible role in stress tolerance inside the host. However, macrophage infection study showed 


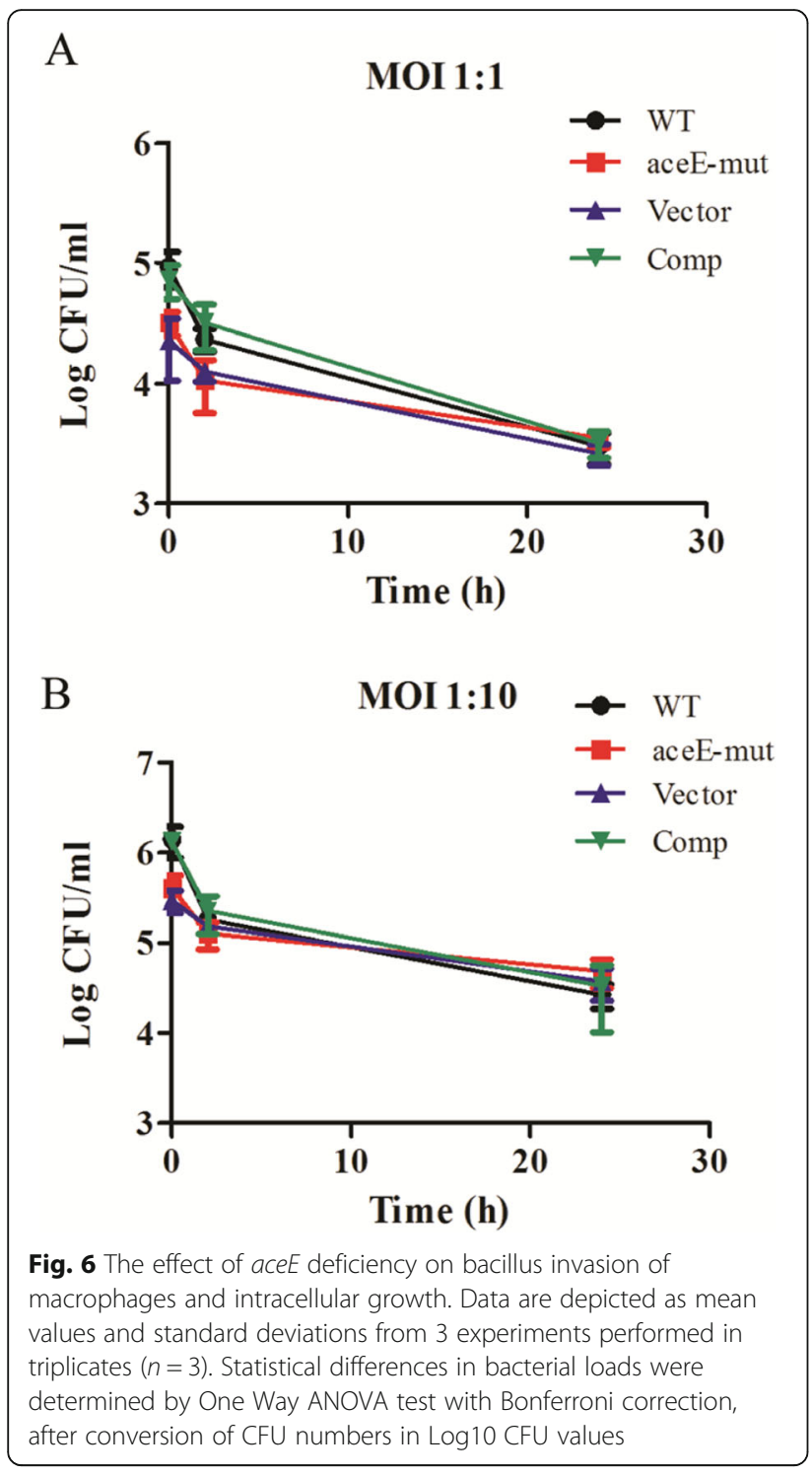

that the inactivation of aceE gene in $M$. smegmatis does not affect bacterial proliferation in macrophages, but rather affects the ability of $M$. smegmatis to invade the macrophages. Therefore, we suggest that the aceE gene is a virulence factor of $M$. smegmatis that may be important in the initiation of infection in vivo. Since M. smegmatis does not have pathogenicity, the study using this bacterium model cannot reveal the possible function of $a c e E$ gene in Mtb pathogenesis. Therefore, the role of aceE gene in pathogenesis requires to be further explored using the virulent Mtb H37Rv strain.

\section{Conclusions}

An aceE mutant $M$. smegmatis $\mathrm{mc}^{2} 155$ strain selected from transposon library presented small, smooth morphology without jagged edges. Compared with its parental WT strain, aceE-mut lost the ability of growing aggregately as well as biofilm formation, and became more fragile to acidic stress. Additionally, alteration of the mycolic acid profile in aceE-mut may directly impact the overall cell wall morphology and acid sensitivity. All these changes of the mutant strain demonstrate that ace $E$ gene inactivation reduces biosynthesis of $\alpha-\mathrm{MA}$, affects the integrity of mycobacterial cell wall, and decreases invasion of macrophage. Since acetyl-coA is an important precursor for the biosynthesis of mycolic acid, we hypothesized that the inactivation of aceE gene may lead to the restriction of acetyl-coA synthesis, thus affecting the biosynthesis of certain mycolic acids. Further study should be conducted to address this issue.

\section{Methods}

Strains, medium, condition

A transposon library was generated using $M$. smegmatis $\mathrm{mc}^{2} 155$ as previously described $[27,28]$ and plated on 7H10 agar containing $20 \mathrm{mg} / \mathrm{L}$ kanamycin. Approximately, 1000 single colonies of variable sizes were randomly placed into 96 deep well plates containing 0.5 $\mathrm{mL}$ of Middlebrook 7H9 medium (BD Difco) containing kanamycin and grown at $37^{\circ} \mathrm{C}$. After 5 days of growth, cultures from each well were spotted on the Middlebrook 7H10 agar (BD Difco) plates, and more than 6 mutants were found for colony morphology defects. $E$. coli strain DH5 $\alpha$ pir 116 (kindly provided by Dr. Kaixia $\mathrm{Mi})$ was used to identify the insertion site of the transposon mutant. E. coli strain Top10 (TransGen Biotech, China) was used to clone specific DNA fragments into pSMT3 plasmid (Table 1). When required, kanamycin (50 mg/L for $E$. coli and $20 \mathrm{mg} / \mathrm{L}$ for mycobacteria) and hygromycin $(150 \mathrm{mg} / \mathrm{L}$ for $E$. coli and $75 \mathrm{mg} / \mathrm{L}$ for mycobacteria) were added to the growth medium.

\section{Transposon identification}

To identify mutants with growth defects, genomic DNA was prepared from the selected transposon mutant. The genomic DNA was randomly digested with BamHI (Fermentas International Inc.) and then purified with a DNA extraction kit (Fermentas International Inc.). The purified DNA was ligated and transformed into DH5 $\alpha$ pir116 competent cells. The plasmids from the kanamycinselected positive colony were isolated and sequenced with following primers: TLP1 5 '-GCTGACCGCTTCCTCGTG CTTTA-3'; TLP2 5'-GCAGCGCATCGCCTTCTATC-3'.

\section{Construction of complemented strain of aceE-Mut}

For complementation of aceE-mut strain, $2.79 \mathrm{~kb}$ fulllength aceE gene (MSMEG_4323) from M. smegmatis was cloned into the mycobacterial shuttle vector pSMT3 [30] using NEBuilding pfu kit (New England Biolabs, Ipswich, MA), and pSMT3-aceE was generated (Table 1). The plasmid pSMT3-aceE was subsequently transformed into 


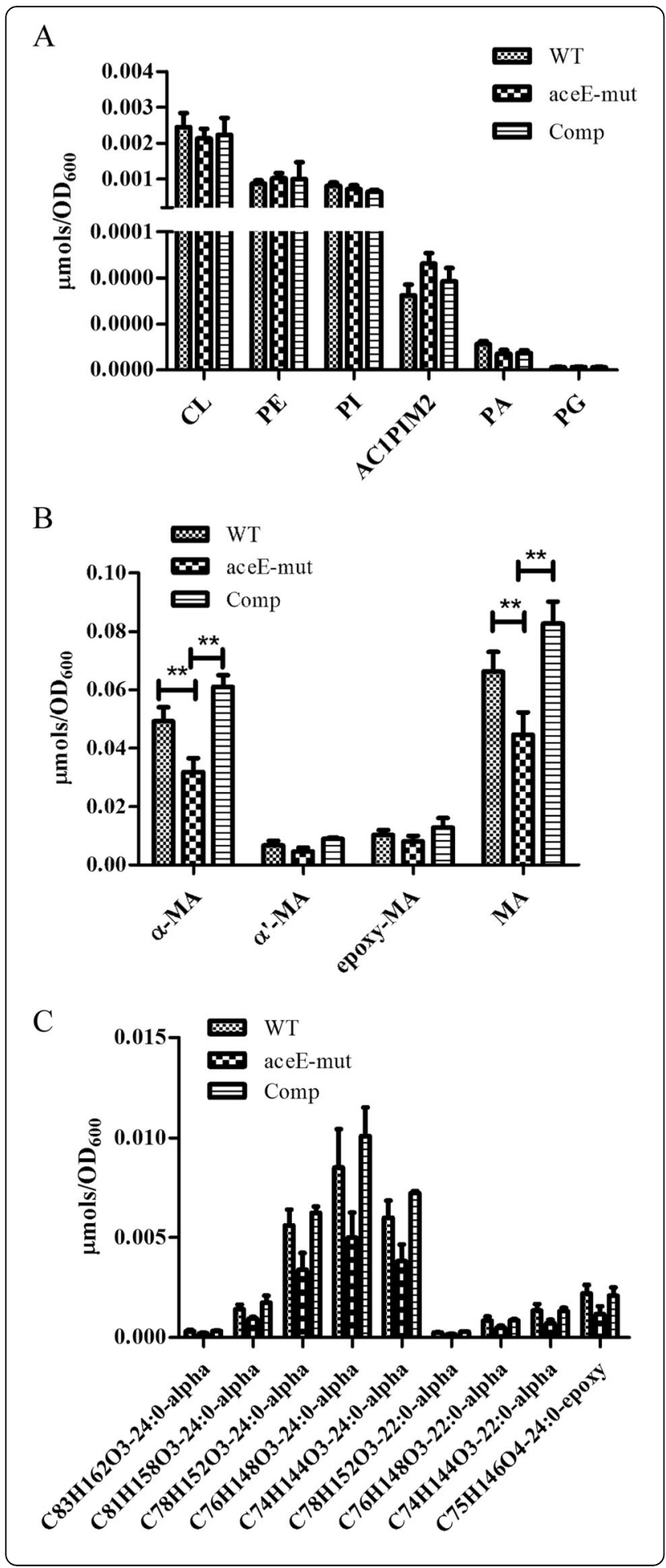

Fig. 7 The effect of aceE deficiency on lipids composition of mycobacterium. Lipids were quantitated by LC-MS. a. The lipids composition other than mycolic acid of WT, aceE-mut and Comp strain; $\mathbf{b}$. The mycolic acid composition of WT, aceE-mut and Comp strain. (**, $P<0.01)$; c. Only the components with significant difference in expression between aceE-mut and WT [and complementary (Comp)] strains were shown in the diagram (One Way ANOVA with Tukey's HSD correction). Data are depicted as mean values and standard deviations from one experiment performed in quadruplicate $(n=4) . \mathrm{CL}$, cardiolipins; $\mathrm{PE}$, phosphatidylethanolamines; PI, phosphatidylinositols; AC1PIM2, Monoacylated Phosphatidylinositol Dimannoside; PA, phosphatidic acids; PG, phosphatidylglycerols; MA, mycolic acids

aceE-mut strain to generate the complemented strain i.e. Comp (Table 1). The transformants were selected on 7H10 agar plates, supplemented with $20 \mathrm{mg} / \mathrm{L}$ kanamycin and $75 \mathrm{mg} / \mathrm{L}$ hygromycin, followed by incubation at $37^{\circ} \mathrm{C}$ for 3-4 days. The positively grown colonies were picked and identified by PCR-sequencing methods using following primers:

aceE_S-FP1 5'-CGGGCTGCAGGAATTCGATTTGACCACCGAGTTCG-3'

aceE_S-RP1 5'-GACGGTATCGATAAGCTTGATTCAGGCGCTGCCGGTG-3'

\section{Colony morphology observation}

To compare the colony sizes for different mycobacterial strains, log phase cultures were $10 \times$ serially diluted (1:10), grown on $7 \mathrm{H} 10$ medium at $37^{\circ} \mathrm{C}$ and examined visually for any change. Photographs were taken after 3-4 days of incubation using stereo microscope (Leica MZ APO).

\section{Morphological observation by electron microscopy}

Mycobacteria from log phase were harvested and washed with $0.1 \mathrm{M}$ phosphate buffer (PBS). Cells were subsequently fixed using $2.5 \%$ glutaraldehyde. Post fixation was carried out in $1 \%$ osmic acid. Following several rinses with $\mathrm{ddH}_{2} \mathrm{O}$, samples were dehydrated in a series of different concentrations of ethanol and $100 \%$ acetone. For transition solvent, resin: acetone (2:1) were used overnight. Epoxy resin-812 was used for $1 \mathrm{~h}$ for embedding. $90 \mathrm{~nm}$ sections were cut and stained with uranyl acetate and Reynold's lead citrate (Ted Pella, Inc). After drying, transmission electron microscopic (TEM) images of the sections were taken using TEM-1400plus. The cell wall thickness was measured for each strain as follows: 100 mycobacteria in the visual field were randomly selected, the largest distance between the outer membrane and the inner membrane of each cell was measured and the data were statistically analyzed using One Way ANOVA with Bonferroni correction. For scanning electron microscopy (SEM), ethanol dehydrated samples were dried in freeze-drier and coated with $10 \mathrm{~nm}$ gold film 
Table 1 Strains and plasmids used in this study

\begin{tabular}{|c|c|c|}
\hline Strain or plasmid & Relevant characteristic & Source or reference \\
\hline \multicolumn{3}{|l|}{ Strains } \\
\hline M. smegmatis $\mathrm{mc}^{2} 155$ & WT, ATCC19420 & {$[29]$} \\
\hline aceE-mut & $m^{2} 155$ with aceE-mut disrupted by Himar1 transposon & This study \\
\hline Vector & aceE-mut complemented with pSMT3-M plasmid & This study \\
\hline Comp & aceE-mut complemented with pSMT3-aceE & This study \\
\hline \multicolumn{3}{|l|}{ Plasmids } \\
\hline Mar T7 & & {$[28]$} \\
\hline pSMT3 & Carries hyg', E. coli-mycobacterial shuttle vector & {$[30]$} \\
\hline pSMT3-aceE & aceE gene cloned under hsp60 promoter in PSMT3-M vector & This study \\
\hline
\end{tabular}

using ion sputter. Scanning electron microscopic images were taken using HITACHI SU8010.

\section{Estimation of pellicle and biofilm formation}

For pellicle formation assay, mycobacteria were inoculated in $4 \sim 5 \mathrm{~mL}$ of Middlebrook $7 \mathrm{H} 9$ medium without Tween-80 and grown at $37{ }^{\circ} \mathrm{C}$ without shaking. Biofilm formation was measured in M63-based liquid medium as previously described [31-34]. Biofilms of all three strains were grown in 96-well polystyrene plates or glass tubes containing M63-based liquid medium complemented with casein hydrolysate and glucose (without Tween-80), inoculated with $0.1 \% \log$ phase culture, and incubated at $30{ }^{\circ} \mathrm{C}$ for 5-7 days under static conditions $[33,34]$. The biofilm formation in each of the liquid cultures was qualitatively analyzed by photography and the images were processed using Adobe Photoshop CS5 software, and quantified with crystal violet staining, as previously described [31, 32].

\section{Growth profile of strains}

The growth characteristics of $M$. smegmatis $\mathrm{mc}^{2} 155$, aceE-mut, Vector (aceE-mut:Vector) and Comp (aceEmut:aceE) strains were studied in neutral ( $\mathrm{pH} 6.8)$ or acidified ( $\mathrm{pH} 5.0$, the $\mathrm{pH}$ was adjusted with hydrochloric acid) $7 \mathrm{H} 9$ medium. The cultures were inoculated with an initial optical density at $600 \mathrm{~nm}\left(\mathrm{OD}_{600}\right)$ of 0.01 and incubated at $37^{\circ} \mathrm{C}$ with constant shaking at $200 \mathrm{rpm}$. $\mathrm{OD}_{600}$ was measured at specified time intervals and 10fold serial dilutions were plated on $7 \mathrm{H} 10$ agar plates for colony forming unit (CFU) counts.

\section{Stress assays}

To carry out in vitro stress studies, logarithmic phase $M$. smegmatis cultures $\left(\mathrm{OD}_{600} \sim 0.8\right)$ were harvested whereas diluted cultures were subjected to different stresses. For oxidative stress, M. smegmatis cultures at $\mathrm{OD}_{600}(\sim 0.4)$ were exposed to hydrogen peroxide $\left(\mathrm{H}_{2} \mathrm{O}_{2}, 0.1 \%\right.$ or $\left.1 \%\right)$ and CFU was determined after $24 \mathrm{~h}$.
For other stresses, M. smegmatis cultures, prepared as above, were adjusted to $\mathrm{OD}_{600}=0.4$ and $\mathrm{NaNO}_{2}(0.5 \%$ or $5 \%$ ) or Sodium dodecyl sulfonate (SDS, $0.1 \%$ or $1 \%$ ) was added. CFU was determined after $1 \mathrm{~h}$ with SDS and after $24 \mathrm{~h}$ with $\mathrm{NaNO}_{2}$.

\section{Antimicrobial susceptibility testing}

Minimal Inhibitory Concentration (MIC) determination was performed by using the alamar blue microtiter assay as recommended in CLSI guidelines [6]. The antibiotics tested in the study include isoniazid (INH), rifampicin (RFP), ethambutol (EMB), ofloxacin (OFX), levofloxacin (LFX), moxifloxacin (MFX), amikacin (AMK) and capreomycin (CPM). The bacterial suspensions of $1.0 \times$ $10^{6} \mathrm{CFU} /$ well were seeded in 96-well plates in presence of antibiotics at concentrations $0.5,1,2,4,8,16,32,64$, 128 , and $256 \mu \mathrm{g} / \mathrm{mL}$ and incubated at $37^{\circ} \mathrm{C}$ for 2 days. Alamar blue dye was added in each well and the plates were re-incubated at $37^{\circ} \mathrm{C}$ for $24 \mathrm{~h}$. The color change from blue to pink indicated bacterial growth. The MIC was defined as the minimal concentration of the drug showed no color changes, which was the lowest concentration of drug that can inhibit the visible growth of the bacterium.

\section{Mycolic acid analysis using HPLC-Sherlock mycobacterium identification system}

Bacterial cultures were collected for isolation of mycolic acids in the cell walls by extraction, saponification, and derivation according to instructions for the Sherlock Mycobacteria Identification System (SMIS; MIDI, Inc.). Mycolic acid composition of each sample was analyzed by SMIS using HPLC.

\section{Analysis of the composition of mycolic acids and lipids using LC-MS}

Total lipids were extracted from the samples using an improved Bligh/Dyer extraction method (double extraction) and appropriate internal standards were 
added as previously described [16]. Analysis of mycolic acids and lipids was carried out using normal-phase LC-MS as previously described, with minor modification $[35,36]$. The experiments were conducted with the help of Lipidall Technologies Company Limited (Changzhou, Jiangsu, China). Briefly, the Exion uplc-qtrap 6500 PLUS (Sciex) liquid-mass spectrometer was used for all of the experiments whereas the electric spray ionization (ESI) mode was used for all the analyses. The following conditions were used: curtain gas $=20$, ion spray voltage $=5500$ $\mathrm{V}$, temperature $=400^{\circ} \mathrm{C}$, ion source gas $1=35$, and ion source gas $2=35$. Phenomenex Luna $3-\mu \mathrm{m}$ silica column (inner diameter $150 \times 2.0 \mathrm{~mm}$ ) was used to separate different kinds of polar lipids using mobile phase A (chloroform: methanol: ammonia 89.5:10:0.5) and B (chloroform: methanol: ammonia: water 55:39:0.5:5.5) using NP-HPLC. The gradient of mobile phase A was maintained for $5 \mathrm{~min}$ from $95 \%$, then linearly decreased to $60 \%$ within 7 min and maintained for $4 \mathrm{~min}$, and then it was further reduced to $30 \%$ and maintained for $15 \mathrm{~min}$. Finally, the initial gradient was maintained for $5 \mathrm{~min}$. Multiple reactions monitoring (MRM) conversion was established for the comparative analysis of various polar lipids and the signal intensity of each MRM value was normalized to an internal standard for quantitative comparisons.

\section{Macrophage infections}

Bacteria at log phase was collected and washed with RPMI1640 before infection. Infection of THP-1 cell (ATCC TIB-202) was performed at a multiplicity of infection (MOI) of 10:1 and 1:1 (bacteria: macrophage), using the following conversion: an OD of $1=3 \times 10^{8}$ $\mathrm{CFU} / \mathrm{mL}$. After $2 \mathrm{~h}$ of incubation at $37^{\circ} \mathrm{C}$, the extracellular mycobacteria were removed by three washings with $1 \times$ PBS and RPMI1640 complete medium containing $100 \mu \mathrm{g} / \mathrm{ml}$ gentamycin was added to inhibit growth of exogenous mycobacteria in infected wells. At $0 \mathrm{~h}, 2 \mathrm{~h}$ and $24 \mathrm{~h}$, infected macrophages were harvested and lysed with $0.1 \%$ Tween- 80 . Then the lysates were serially diluted with $0.05 \%$ Tween-80, and plated on $7 \mathrm{H} 10$ agar plates with or without the antibiotic. The plates were incubated at $37^{\circ} \mathrm{C}$ until colonies could be counted.

\section{Statistical analysis}

All statistical analyses were performed using SPSS statistics 21. Statistical differences were determined by One Way ANOVA with Bonferroni correction when comparing more than two groups. For mycolic acid and lipid quantification experiments, One Way ANOVA with Tukey's HSD correction was used. Student $t$ test was used to compare the statistical differences between two groups. Only $P$ values $<0.05$ were considered as statistically significant.

\section{Supplementary information}

Supplementary information accompanies this paper at https://doi.org/10. 1186/s12866-020-01940-2.

Additional file 1: Figure S1. Different growth of M. smegmatis $\mathrm{mc}^{2} 155$ (WT) and aceE-mut after treatment with different chemical agents.

Additional file 2: Figure S2. The effect of aceE deficiency on mycolic acid composition in mycobacterium.

Additional file 3: Table S1. MICs for M. smegmatis $\mathrm{mc}^{2} 155$ and aceEmut with different antibiotics.

\section{Abbreviations}

PDH: Pyruvate dehydrogenase complex; aceE-mut: aceE-deficient mutants; WT: Wild-type; MA: Mycolic acid; Mtb: Mycobacterium tuberculosis; antiTB: Anti-tuberculosis; MIC: Minimal Inhibitory Concentration; SMIS: Sherlock Mycobacteria Identification System; ESI: Electric spray ionization; MRM: Multiple reactions monitoring; CFU: Colonies forming unit; SDS: Sodium dodecyl sulfonate; ECL: The Equivalent Carbon Length of the mycolic acid; $\mathrm{H}_{2} \mathrm{O}_{2}$ : Hydrogen peroxide; INH: Isoniazid; RFP: Rifampicin; EMB: Ethambutol; OFX: Ofloxacin; LFX: Levofloxacin; MFX: Moxifloxacin; AMK: Amikacin; CPM: Capreomycin; TEM: Transmission electron microscopy; SEM: Scanning electron microscopy; CL: Cardiolipins;

PE: Phosphatidylethanolamines; PI: Phosphatidylinositols;

AC1PIM2: Monoacylated Phosphatidylinositol Dimannoside; PA: Phosphatidic acids; PG: Phosphatidylglycerols

\section{Acknowledgements}

We thank Prof. Guanghou Shui for his kindly help in lipid data reanalysis and helpful discussions.

Authors' contributions

STC and HRH conceived and designed the experiments. STC, TLT, SAW, and TTZ performed the experiments. STC analyzed the data and was the major contributor in writing the manuscript. All authors have read and approved the manuscript.

\section{Funding}

The study was supported by National Natural Science Foundation of China (No. 31600107 and No. 81672065), Beijing Natural Science Foundation (No.5192006), National Major Science and Technology Projects of China (2018ZX10302-301-004), Tong Zhou "Yun He" Talent Project (YHLD2018030), Beijing Municipal Administration of Hospitals' Ascent Plan (DFL20181602), and Beijing Municipal Administration of Hospitals Clinical Medicine Development of Special Funding Support (ZYLX201809). The funding sources were not involved in study design, sample collection, data analysis and interpretation

\section{Availability of data and materials}

All data generated or analysed during this study are included in this published article and its supplementary information files.

Ethics approval and consent to participate Not applicable.

Consent for publication Not applicable.

\section{Competing interests}

The authors declare that they have no competing interests.

Received: 16 April 2020 Accepted: 11 August 2020

Published online: 18 August 2020

References

1. Schick J, Etschel P, Bailo R, Ott L, Bhatt A, Lepenies B, et al. Toll-like receptor 2 and Mincle cooperatively sense Corynebacterial Cell Wall glycolipids. Infect Immun. 2017;85(7):e00075. https://doi.org/10.1128/IAI.00075-17. 
2. Awuh JA, Flo TH. Molecular basis of mycobacterial survival in macrophages. Cell Mol Life Sci. 2017;74(9):1625-48. https://doi.org/10.1007/s00018-0162422-8.

3. Chatterjee D. The mycobacterial cell wall: structure, biosynthesis and sites of drug action. Curr Opin Chem Biol. 1997;1(4):579-88 doi: S13675931(97)80055-5 [pii].

4. Daffe M, Draper P. The envelope layers of mycobacteria with reference to their pathogenicity. Adv Microb Physiol. 1998;39:131-203.

5. Brennan PJ. Structure, function, and biogenesis of the cell wall of mycobacterium tuberculosis. Tuberculosis. 2003;83(1-3):91-7. https://doi. org/10.1016/s1472-9792(02)00089-6

6. Clinical and Laboratory Standards Institute, editor. Susceptiblity testing of mycobacteria, Nocardiae, and other aerobic Actinomycetes; approved standard. 2nd ed: Clinical and Laboratory Standards Institute; 2011.

7. Martin E, Rosenthal RE, Fiskum G. Pyruvate dehydrogenase complex: metabolic link to ischemic brain injury and target of oxidative stress. J Neurosci Res. 2005;79(1-2):240-7. https://doi.org/10.1002/jnr.20293.

8. Shimakata T, Fujita Y, Kusaka T. Acetyl-CoA-dependent elongation of fatty acids in mycobacterium smegmatis. J Biochem. 1977;82(3):725-32. https:// doi.org/10.1093/oxfordjournals.jbchem.a131749.

9. Shimakata T, Fujita Y, Kusaka T. Involvement of one of two enoyl-CoA hydratases and enoyl-CoA reductase in the acetyl-CoA-dependent elongation of medium chain fatty acids by mycobacterium smegmatis. $J$ Biochem. 1980;88(4):1051-8. https://doi.org/10.1093/oxfordjournals.jbchem. a133056.

10. Schnappinger D, Ehrt S, Voskuil MI, Liu Y, Mangan JA, Monahan IM, et al. Transcriptional adaptation of mycobacterium tuberculosis within macrophages: insights into the Phagosomal environment. J Exp Med. 2003; 198(5):693-704. https://doi.org/10.1084/jem.20030846 jem.20030846 [pii].

11. Timm J, Post FA, Bekker LG, Walther GB, Wainwright HC, Manganelli R, et al. Differential expression of iron-, carbon-, and oxygen-responsive mycobacterial genes in the lungs of chronically infected mice and tuberculosis patients. Proc Natl Acad Sci U S A. 2003;100(24):14321-6. https://doi.org/10.1073/pnas.2436197100 2436197100 [pii].

12. Cole ST, Brosch R, Parkhill J, Garnier T, Churcher C, Harris D, et al. Deciphering the biology of mycobacterium tuberculosis from the complete genome sequence. Nature. 1998;393(6685):537-44. https://doi.org/10.1038/ 31159 .

13. Schreiner ME, Fiur D, Holatko J, Patek M, Eikmanns BJ. E1 enzyme of the pyruvate dehydrogenase complex in Corynebacterium glutamicum: molecular analysis of the gene and phylogenetic aspects. J Bacteriol. 2005; 187(17):6005-18. https://doi.org/10.1128/JB.187.17.6005-6018.2005.

14. Shi S, Ehrt S. Dihydrolipoamide acyltransferase is critical for Mycobacterium tuberculosis pathogenesis. Infect Immun. 2006;74(1):56-63. https://doi.org/10 1128/IAl.74.1.56-63.2006.

15. Bryk R, Gold B, Venugopal A, Singh J, Samy R, Pupek K, et al. Selective killing of nonreplicating mycobacteria. Cell Host Microbe. 2008;3(3):137-45. https:// doi.org/10.1016/j.chom.2008.02.003.

16. Venugopal A, Bryk R, Shi S, Rhee K, Rath P, Schnappinger D, et al. Virulence of mycobacterium tuberculosis depends on lipoamide dehydrogenase, a member of three multienzyme complexes. Cell Host Microbe. 2011;9(1):2131. https://doi.org/10.1016/j.chom.2010.12.004 S1931-3128(10)00414-2 [pii].

17. Tian J, Bryk R, Shi S, Erdjument-Bromage H, Tempst P, Nathan C. Mycobacterium tuberculosis appears to lack alpha-ketoglutarate dehydrogenase and encodes pyruvate dehydrogenase in widely separated genes. Mol Microbiol. 2005;57(3):859-68doi: MMI4741 [pii]. https://doi.org/ 10.1111/j.1365-2958.2005.04741.x

18. Li AH, Lam WL, Stokes RW. Characterization of genes differentially expressed within macrophages by virulent and attenuated mycobacterium tuberculosis identifies candidate genes involved in intracellular growth. Microbiology. 2008;154(Pt 8):2291-303. https://doi.org/10.1099/mic.0.2008/ 019661-0 154/8/2291.

19. Manganelli R, Voskuil MI, Schoolnik GK, Smith I. The mycobacterium tuberculosis ECF sigma factor sigmaE: role in global gene expression and survival in macrophages. Mol Microbiol. 2001;41(2):423-37 doi: mmi2525 [pii].

20. Heplar JQ, Clifton CE, Raffel S, Futrelle CM. Virulence of the tubercle bacillus. I. Effect of oxygen tension upon respiration of virulent and avirulent bacilli. J Infect Dis. 1954:94(1):90-8.

21. Murthy PS, Sirsi M, Ramakrishnan T. Tricarboxylic acid cycle and related enzymes in cell-free extracts of mycobacterium tuberculosis H37Rv. Biochem J. 1962;84:263-9.
22. Maksymiuk C, Balakrishnan A, Bryk R, Rhee KY, Nathan CF. E1 of alphaketoglutarate dehydrogenase defends mycobacterium tuberculosis against glutamate anaplerosis and nitroxidative stress. P Natl Acad Sci USA. 2015; 112(43):E5834-E43. https://doi.org/10.1073/pnas.1510932112.

23. Viswanathan G, Joshi SV, Sridhar A, Dutta S, Raghunand TR. Identifying novel mycobacterial stress associated genes using a random mutagenesis screen in mycobacterium smegmatis. Gene. 2015;574(1):20-7. https://doi. org/10.1016/j.gene.2015.07.063.

24. Jamet S, Slama N, Domingues J, Laval F, Texier P, Eynard N, et al. The nonessential mycolic acid biosynthesis genes hadA and hadC contribute to the physiology and fitness of mycobacterium smegmatis. PLoS One. 2015. 10(12):e0145883. https://doi.org/10.1371/journal.pone.0145883.

25. Singh A, Varela C, Bhatt K, Veerapen N, Lee OY, Wu HH, et al. Identification of a desaturase involved in mycolic acid biosynthesis in mycobacterium smegmatis. PLoS One. 2016;11(10):e0164253. https://doi.org/10.1371/journal. pone. 0164253

26. Lefebvre C, Boulon R, Ducoux M, Gavalda S, Laval F, Jamet S, et al. HadD, a novel fatty acid synthase type II protein, is essential for alpha- and epoxymycolic acid biosynthesis and mycobacterial fitness. Sci Rep. 2018;8(1):6034. https://doi.org/10.1038/s41598-018-24380-5.

27. Sassetti CM, Boyd DH, Rubin EJ. Comprehensive identification of conditionally essential genes in mycobacteria. P Natl Acad Sci USA. 2001; 98(22):12712-7. https://doi.org/10.1073/pnas.231275498.

28. Bardarov SS, Bardarov SS Jr, Jacobs WR Jr. Transposon mutagenesis in mycobacteria using conditionally replicating mycobacteriophages. Methods Mol Med. 2001;54:43-57. https://doi.org/10.1385/1-59259-147-7:043.

29. Snapper SB, Melton RE, Mustafa S, Kieser T, Jacobs WR Jr. Isolation and characterization of efficient plasmid transformation mutants of mycobacterium smegmatis. Mol Microbiol. 1990;4(11):1911-9. https://doi. org/10.1111/j.1365-2958.1990.tb02040.x.

30. Golanska E, Brzostek A, Kiatpapan P, Dziadek J. Characterisation of a new host-vector system for fast-growing mycobacteria. Acta Microbiol Pol. 1998; 47(4):335-43.

31. Recht J, Kolter R. Glycopeptidolipid acetylation affects sliding motility and biofilm formation in mycobacterium smegmatis. J Bacteriol. 2001;183(19): 5718-24. https://doi.org/10.1128/Jb.183.19.5718-5724.2001.

32. Recht J, Martinez A, Torello S, Kolter R. Genetic analysis of sliding motility in mycobacterium smegmatis. J Bacteriol. 2000;182(15):4348-51. https://doi. org/10.1128/Jb.182.15.4348-4351.2000

33. Mycobacteria protocols, third edition. In. Edited by Roberts TPaDM, Third edn. New York: Springer Science; 2015.

34. Ojha A, Anand M, Bhatt A, Kremer L, Jacobs WR Jr, Hatfull GF. GroEL1: a dedicated chaperone involved in mycolic acid biosynthesis during biofilm formation in mycobacteria. Cell. 2005;123(5):861-73. https://doi.org/10.1016/ j.cell.2005.09.012.

35. Lam SM, Tong L, Duan XR, Petznick A, Wenk MR, Shui GH. Extensive characterization of human tear fluid collected using different techniques unravels the presence of novel lipid amphiphiles. J Lipid Res. 2014;55(2): 289-98. https://doi.org/10.1194/jlr.M044826.

36. Gong H, Li J, Xu A, Tang Y, Ji W, Gao R, et al. An electron transfer path connects subunits of a mycobacterial respiratory supercomplex. Science. 2018;362:6418. https://doi.org/10.1126/science.aat8923.

\section{Publisher's Note}

Springer Nature remains neutral with regard to jurisdictional claims in published maps and institutional affiliations.

Ready to submit your research? Choose BMC and benefit from:

- fast, convenient online submission

- thorough peer review by experienced researchers in your field

- rapid publication on acceptance

- support for research data, including large and complex data types

- gold Open Access which fosters wider collaboration and increased citations

- maximum visibility for your research: over $100 \mathrm{M}$ website views per year

At $\mathrm{BMC}$, research is always in progress.

Learn more biomedcentral.com/submissions 